\title{
Localization and Synthesis of Prenylquinones in Isolated Outer and Inner Envelope Membranes from Spinach Chloroplasts
}

\author{
JÜRGEN SOLL,,* GERNOT SCHULTZ, $†$ JACQUES JOYARD, $\ddagger$ ROLAND DOUCE,,$\ddagger$ \\ AND MARYSE A. BLOCK $\ddagger$
}

\author{
*Botanisches Institut, Universität München, Menzinger Strasse 67, 8000 München 19, West Germany; \\ $\dagger$ Botanisches Institute, Tierärztliche Hochschule, Bünteweg 17, 3000 Hannover, West Germany; and \\ $\ddagger U A$ CNRS $n^{\circ} 576$, Departement de Recherche Fondamentale, Laboratoire de Physiologie \\ Cellulaire Végétale, Centre d'Etudes Nucléaires et Université Scientifique et \\ Médicale de Grenoble, 85 X, 38041 Grenoble-Cedex, France
}

Received September 18, 1984, and in revised form November 27, 1984

The prenylquinone content and biosynthetic capabilities of membrane fractions enriched in outer and inner envelope membranes from spinach chloroplasts were analyzed. Both envelope membranes contain prenylquinones, and in almost similar amounts (on a protein basis). However, the outer envelope membrane contains more $\alpha$-tocopherol than the inner one although this prenylquinone is the major one in both fractions. On the contrary, plastoquinone- 9 is present in higher amounts in the inner envelope membrane than in the outer one. In addition, it has been demonstrated that all the enzymes involved in the last steps of $\alpha$-tocopherol and plastoquinone-9 biosynthesis, i.e., homogentisate decarboxylase polyprenyltransferase, $S$-adenosyl-methionine:methyl-6-phytylquinol methyltransferase, $S$-adenosyl-methionine: $\alpha$-tocopherol methyltransferase, homogentisate decarboxylase solanesyltransferase, $S$-adenosyl-methionine:methyl-6-solanesylquinol methyltransferase, and possibly 2,3dimethylphytylquinol cyclase, are localized on the inner envelope membrane. These results demonstrate that the inner membrane of the chloroplast envelope plays a key role in chloroplast biogenesis, and especially for the synthesis of the two major plastid prenylquinones. 1985 Academic Press, Ine.

The chloroplast envelope of spinach leaves contains several prenylquinones as basic constituents: plastoquinone-9, phylloquinone, $\alpha$-tocoquinone, and $\alpha$-tocopherol $(1,2)$. Qualitatively, the prenylquinones of both types of chloroplast membranes (thylakoids and envelope membranes) are identical, but the proportions in which they are present are different. Thus plastoquinone-9, a well-known electron carrier between the two photosystems, accounts for a higher proportion of the thylakoid prenylquinone content when compared with the envelope fraction. On the other hand, $\alpha$-tocopherol accounts

\footnotetext{
'To whom correspondence should be addressed.
}

for a higher proportion of the envelope prenylquinone content when compared with the thylakoids. These differences may be characterized by the $\alpha$-tocopherol to plastoquinone-9 ratio, which is much higher in the envelope fraction (2.6) than in the thylakoids (0.3). The minor quinone components ( $\alpha$-locoquinune and phylloquinone) appear in both membrane fractions in similar proportions. In addition, plastohydroquinone- 9 , the reduced form of plastoquinone-9 which occurs in thylakoids at 30 to $50 \%$ of the concentration of plastoquinone, was not detected in envelope membranes $(1,2)$.

It is now well established that the isoprenoid side chains of the terpenic compounds present in plastids are synthesized 
within the organelle (2-4). Indeed, Soll et al. $(5,6)$ have demonstrated that the chloroplast envelope isolated from intact, purified spinach chloroplasts was the only site of $\alpha$-tocopherol biosynthesis [this compound represents more than $40 \%$ of the total prenylquinone content of spinach chloroplasts, (1)]. Thus the enzymes involved in the prenylation of homogentisic acid (homogentisate decarboxylasepolyprenyltransferase) and the following methylation steps of the resulting methyl6-prenylquinol with $S$-adenosyl-methionine are specifically localized in the envelope membranes $(5,6)$. Likewise, Soll et al. (6) have also shown that the chloroplast envelope contains the complete array of plastoquinone biosynthetic machinery. Thus, we have demonstrated that the chloroplast envelope is the only site of prenylation of homogentisic acid with a nonaprenyl pyrophosphate (solanesyl pyrophosphate), yielding 2-methyl-6-solanesylquinol. The envelope membranes also contain the enzyme involved in the following methylation step with $S$-adenosyl-methionine as methyl donor to yield plastoquinol-9 (6).

However, the plastid envelope consists of two morphologically and topologically distinct membranes (7). Therefore, a better understanding of the function of each envelope membrane in terpenoid metabolism requires detailed analyses of the distribution and the precise site of synthesis of prenylquinones within the two envelope membranes. This is the purpose of this article.

\section{EXPERIMENTAL PROCEDURES}

\section{Isolation of Purified Intact Spinach Chloroplasts}

Chloroplasts were isolated from 3-4 kg of spinach leaves obtained from Grenoble farms or local markets. Deveined leaf sections were cut into chilled medium (2.5 liters $/ \mathrm{kg}$ leaves) containing $330 \mathrm{~mm}$ mannitol, $30 \mathrm{mM} \mathrm{Mops,}{ }^{2} 0.1 \%$ bovine serum albumin;

\footnotetext{
${ }^{2}$ Abbreviations used: Mops, 4-morpholinepropanesulfonic acid; Tricine, $N$-[2-hydroxy-1,1-bis(hydroxymethyl)ethyl.glycine.
}

$\mathrm{pH}$ was adjusted to 7.8 with $\mathrm{NaOH}$. The leaves were homogenized in three successive batches for $2-3 \mathrm{~s}$ in a 4-liter Waring blendor. All the operations were carried out in a cold room or on ice. A crude chloroplast pellet was obtained as described by Douce et al. (8) and purified further by isopycnic centrifugation in Percoll gradients (9). The purified intact chloroplasts (150-200 mg chlorophyll, $2.3-3 \mathrm{~g}$ protein) were then used for the preparation of membrane fractions enriched in outer or inner envelope membranes (see below).

\section{Preparation of Membrane Fractions Enriched in Outer and Inner Envelope Membranes}

Intact purified spinach chloroplasts kept in a hypertonic medium $(0.6 \mathrm{M}$ mannitol) were used as described by Block et al. (10). The separation was achieved by applying low pressure to the shrunken chloroplasts and slowly extruding them through the small aperture of a Yeda press under controlled conditions (10). By centrifugation of the mixture at $12,000 \mathrm{~g}$ for $10 \mathrm{~min}$, a greenish supernatant was obtained which was then layered on top of a discontinuous sucrose gradient, as described by Block et al. (10). The two membrane fractions obtained were characterized as described by Block et al. $(10,11)$ using electrophoretic and immunochemical analyses and the distribution of marker enzymes. Indeed, the light fraction $\left(\mathrm{d}-1.08 \mathrm{~g} / \mathrm{cm}^{3}\right)$ obtained at the 0.4 / $0.65 \mathrm{M}$ sucrose interface, contained more than $90 \%$ outer envelope membrane (on a protein basis), whereas the heavy fraction $\left(\mathrm{d}=1.13 \mathrm{~g} / \mathrm{cm}^{3}\right)$, obtained at the $0.65 / 1 \mathrm{M}$ sucrose interface, contained about $80 \%$ of inner envelope membrane protein. In addition, each membrane fraction has a characteristic pigment and polar lipid composition (11) and contained specific enzymes such as acyl-CoA synthetase in the outer envelope membrane (12) and the enzymes involved in galactolipid biosynthesis in the inner envelope membrane $(11,13)$. Therefore, the two membrane fractions obtained according to Block et al. (10) have been thoroughly identified (10-13).

\section{Determination of Prenylquinones in Membrane Fractions Enriched in Outer and Inner Envelope Membranes}

The prenylquinone content of the membrane fractions obtained was determined using high-pressure liquid chromatography. The envelope prenylquinones were extracted from deep-frozen preparations according to the method of Bligh and Dyer (14). The chloroformic phase was evaporated to dryness under a stream of argon. The residue was suspended in ethanol and used for prenylquinone analyses as described by Lichtenthaler et al. (1). Prenylquinone 
separation was achieved using a reversed-phase column (RPy, 7- $\mu \mathrm{m}$ mesh; Merck) and methanol:water (95.7:4.3, v/v) as developing solvent (flow rate, 1.5 $\mathrm{ml} / \mathrm{min}$ ). Quinones, quinols, and $\alpha$-tocopherol were detected in the same run using two UV detectors set at 250 and $292 \mathrm{~nm}$, respectively. The components were identified by their retention times, which were compared with those of reference substances. In addition, in some runs, the reference substances were injected together with the samples for further characterization. The extinction coefficients used for quantitative calculations were taken from Threlfall and Goodwin (15).

\section{Assays of $\alpha$-Tocopherol and Plastoquinone-9 Synthesis}

Chemicals. $S-\left[\right.$ methyl $\left.{ }^{14} \mathrm{C}\right]$ Adenosyl-methionine (sp act, $46 \mathrm{mCi} / \mathrm{mmol}$ ) was purchased from CEA (Saclay, France); $\left[{ }^{3} \mathrm{H}\right]$ homogentisic acid was labeled by catalyst-mediated tritium exchange (CEA tritium exchange service, Saclay, France) and purified prior to use by thin-layer chromatography on precoated silica gel plates (G 1500 LS254, Schleicher and Schüll, F. R. Germany) using benzene:dioxane:acetic acid $(50: 45: 4, \mathrm{v} / \mathrm{v})$ as developing solvent. Unlabeled homogentisic acid was added to the tritium-labeled mixture prior to chromatography and the purified $\left[{ }^{3} \mathrm{H}\right]$ homogentisic acid thus obtained had a specific activity of $500 \mathrm{mCi} / \mathrm{mmol}$ and was stored in ethanol. Phytylpyrophosphate (phytyl-PP) and solanesyl pyrophosphate (solanesyl-PP) were synthesized from the corresponding alcohols and triethylammoniumphosphate as described by Joo et al. (16). Methyl-6phytylquinone and 2,3-dimethylphytylquinone were synthesized as described by Mayer and Isler (17) and Soll and Schultz (18). Methyl-6-phytylquinone $\left(\lambda_{\max }, 252.3 \mathrm{~nm}\right.$; cyclohexane; Zeiss PMQ 3) was purified from methyl-3-phytylquinone and methyl5 -phytylquinone $\left(\lambda_{\max }, 251.5 \mathrm{~nm}\right)$ by multiple rechromatography. Plastoquinone- 9 and $\gamma$-tocopherol were a gift of Dr. F. Weber, Hoffmann-La Roche, Basel, Switzerland.

Reaction mixtures. Standard incubation mixtures (final volume, $0.5 \mathrm{ml}$ ) contained $50 \mathrm{mM}$ Tricine$\mathrm{NaOH}$ (pH 7.7), $4 \mathrm{~mm} \mathrm{MgCl}_{2}$, and the appropriate substrates: $\left[{ }^{8} \mathrm{H}\right]$ homogentisic acid $(60 \mu \mathrm{M})$, phytyl-PP $(0.1 \mathrm{mM})$, solanesyl-PP $(0.1 \mathrm{mM}), S-\left[\right.$ methyl $\left.{ }^{14} \mathrm{C}\right] \mathrm{ade}-$ nosyl-methionine $(0.1 \mathrm{mM})$, methyl-6-phytylquinol $(0.2 \mathrm{mM})$, or $\gamma$-tocopherol $(0.2 \mathrm{mM})$. Prenylquinols were used immediately after reduction with $\mathrm{NaBH}_{4}$ of the corresponding quinone as described by Soll and Schultz (18). The reaction was initiated by addition of the envelope membrane vesicles. Time course experiments were carried out at $20^{\circ} \mathrm{C}$ in the dark. The reaction was stopped and the products extracted by addition of chloroform:methanol (1:2, $\mathrm{v} / \mathrm{v}$ ) to the mixture (14).
Purification of the labeled products. The chloroformic phase obtained as described above was evaporated to dryness under a stream of argon and resuspended in ethanol. A $25-\mu \mathrm{g}$ aliquot of standard substances, corresponding to the expected reaction products, was added to the mixture as carrier, in order to facilitate identification of the substances which were separated by thin-layer chromatography as described by Soll et al (6). The prenylquinols were oxidized by air prior to the purification as described by Soll et al. (6). Purification of the labeled products was done by thin-layer chromatography on silica gel-precoated plates (Schleicher and Schüll) using petroleum ether (bp $\left.40-60^{\circ} \mathrm{C}\right)$ :diethylether $(15$ : $1, \mathrm{v} / \mathrm{v})$ as developing solvent. The components were identified by comparing their $R_{f}$ values with those of reference standards. Tocopherols and quinones were rechromatographed on precoated cellulose plates impregnated with $7 \%$ paraffin, using acetone: water $(85: 15, v / v$, for tocopherols or $95: 5, v / v$, for quinones) as solvent systems (6). Localization of the radioactive substances was also verified by autoradiography using X-Omat AR films (Kodak). The spots were scrapped off the plates, dissolved in $2 \mathrm{ml}$ methanol and mixed with $10 \mathrm{ml}$ scintillation cocktail (Aquasol, Amersham). The radioactivity was determined using an Intertechnique SL 4000 liquid scintillation counter.

\section{Other Assays}

Chlorophyll was determined spectrophotometrically in $80 \%$ acetone extracts of membranes (19). Protein content of each membrane fraction was determined using bovine serum albumin as standard (20).

\section{RESULTS}

\section{Localization of Prenylquinones within Spinach Chloroplast Envelope Membranes}

Lipid extracts of the two envelope membrane fractions exhibit light $a b-$ sorption in the UV range $230-300 \mathrm{~nm}$; the characteristic absorption maxima of prenylquinones in this region facilitated the HPLC analyses. As shown in Table I, both membrane fractions contained prenylquinones, and in almost similar amounts (10 to $12 \mu \mathrm{g} / \mathrm{mg}$ protein). The major prenylquinone in both membrane fractions was $\alpha$-tocopherol, but the outer envelope membrane contained (on a protein basis) $50 \%$ more $\alpha$-tocopherol than the inner envelope membrane. Phylloquinone concentration was in the range of detectabil- 
TABLE I

Distribution of Prenylquinones between Two Membrane Fractions EnRIChed in OUTER OR INNER ENVFLOPF MEMBRANF FROM SPINACH CHLOROPLASTS

\begin{tabular}{llll}
\hline \multicolumn{1}{c}{ Prenylquinone } & $\begin{array}{c}\text { Inner } \\
\text { envelope } \\
\text { fraction }\end{array}$ & $\begin{array}{c}\text { Outer } \\
\text { envelope } \\
\text { fraction }\end{array}$ \\
\hline $\begin{array}{l}\alpha \text {-Tocopherol } \\
\text { Phylloquinone }\end{array}$ & $6.7 \pm 0.3$ & $9.8 \pm 0.6$ \\
Plastoquinone-9 & $0.07 \pm 0.015$ & $0.055 \pm 0.012$ \\
Plastoquinol-9 & $1.63 \pm 0.15$ & $1.1 \quad \pm 0.16$ \\
Total plastoquinones & $1.54 \pm 0.17$ & $1.1 \quad \pm 0.16$ \\
Total prenylquinones & $10.0 \pm 0.7$ & $12.1 \quad \pm 0.9$ \\
\hline
\end{tabular}

Note. The different membrane fractions analyzed were prepared according to the procedure described by Block $e t$ al (10) (see Experimental Procedures). Prenylquinones were extracted, analyzed, and characterized as described in the text. The data given below are mean values of at least five IIPLC determinations. They are expressed as micrograms prenylquinones per milligram envelope protein.

ity and seemed to be more equally distributed than $\alpha$-tocopherol. Plastoquinones were enriched in the inner envelope membrane; the $\alpha$-tocopherol to plastoquinone- 9 ratio was 2.1 in the inner envelope membrane and 4.45 in the outer envelope membrane. These values are much higher than those found in thylakoids (0.3) but are in the same range as the values found in total envelope (2.6) [see Ref. (1)]. Interestingly, plastoquinones were found in both their oxidized and reduced forms with a ratio of about 1 to 1 (Table I).

\section{$\alpha$-Tocopherol Synthesis within \\ Envelope Membranes}

The final steps of $\alpha$-tocopherol synthesis, which are summarized in Fig. 1, have been demonstrated in spinach chloroplast envelope membranes $(5,6)$. Using wellcharacterized membrane fractions enriched in outer and inner envelope membranes (10-13), we measured the activities of the enzymes involved in $\alpha$-tocopherol synthesis within chloroplast envelope membranes.

The first enzyme involved in this synthesis, a homogentisate decarboxylase polyprenyltransferase, catalyzes the prenylation of homogentisic acid with
phytyl-PP to yield methyl-6-phytylquinol. As shown in Fig. 2, most of the activity was present in the inner envelope membrane fraction. Little activity was present in the outer membrane fraction and the ratio of methyl-6-phytylquinol synthesis was about 8 to 1 in favor of the inner membrane fraction. From studies with isolated chloroplasts, it is known that the most probable next reaction in $\alpha$-tocopherol synthesis is the methylation of methyl-6-phytylquinol with $S$-adenosylmethionine to form 2,3-dimethylphytylquinol $(3,6,21)$ (Fig. 1). As shown in Fig. 3, we found again that most of the activity of $S$-adenosyl-methionine: methyl-6-phytylquinol methyltransferase was concentrated in the inner envelope membrane fraction.

Cyclization of 2,3-dimethylphytylquinol to form $\gamma$-tocopherol (Fig. 1) is difficult to demonstrate in purified envelope membranes; in previous experiments (5) with total envelope membranes we found that this activity was at the limit of detectability. The same result was obtained here (results not shown). This is not surprising since in isolated intact chloroplasts, this step was shown to be the slowest in $\alpha$-tocopherol synthesis $(2,5)$.

The final step in $\alpha$-tocopherol synthesis is the methylation of $\gamma$-tocopherol by $S$ adenosyl-methionine (Fig. 1). We found that the $S$-adenosyl-methionine: $\gamma$-tocopherol methyltransferase was almost entirely localized in the membrane fraction enriched in inner envelope membranes (Fig. 4).

From all these data, we can conclude that the final steps in $\alpha$-tocopherol synthesis occur in the inner envelope membrane of spinach chloroplasts. The activities found in the membrane fraction enriched in outer envelope are always very low and can be attributed to cross-contamination by small vesicles deriving from the inner membrane [see Ref. (10)].

\section{Plastoquinone-9 Synthesis within Spinach Chloroplast Envelope Membranes}

Two enzymes are involved in the last steps of plastoquinone-9 synthesis (Fig. 


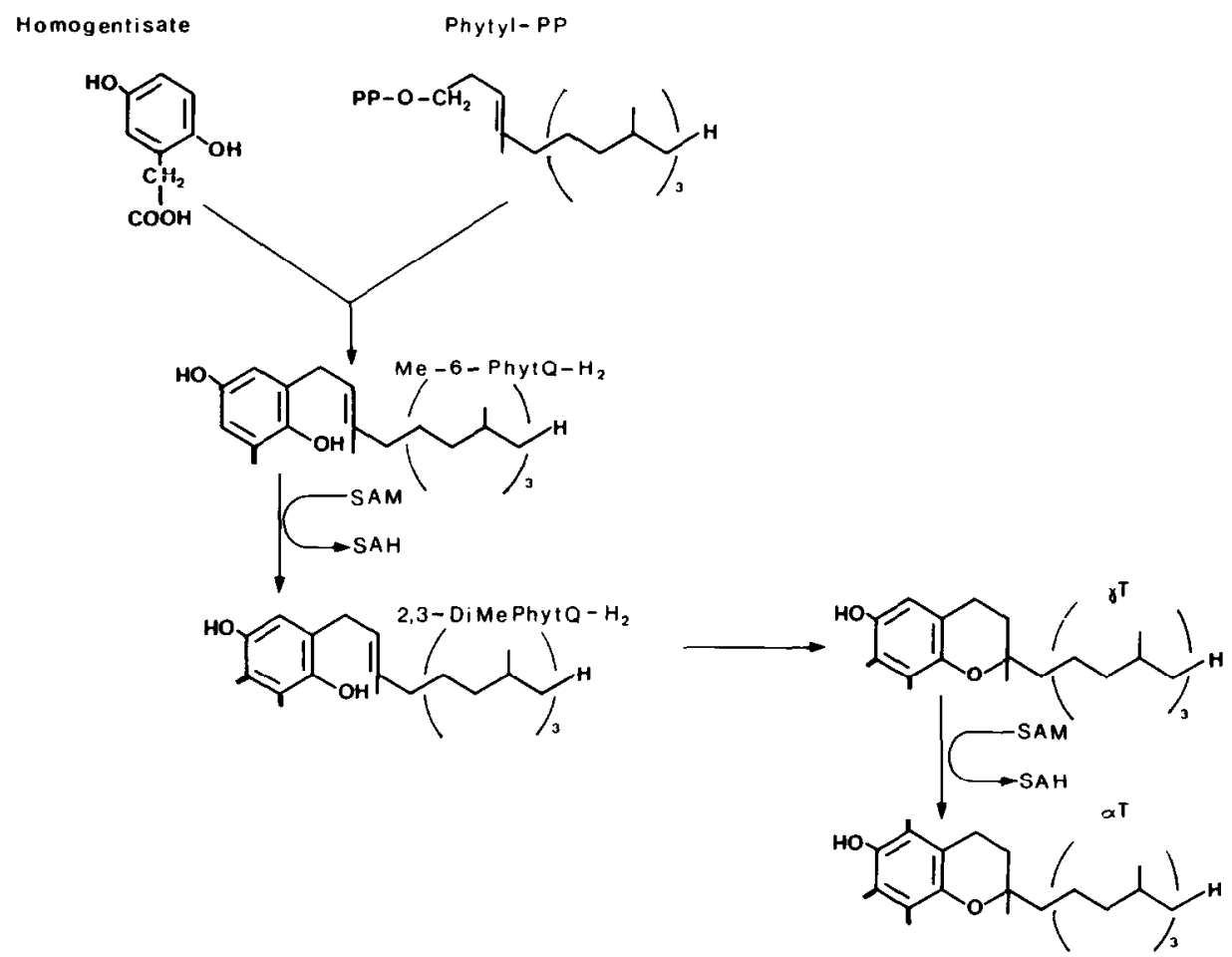

FlG. 1. Summary of the main biosynthetic pathway for $\alpha$-tocopherol biosynthesis in spinach chloroplasts.

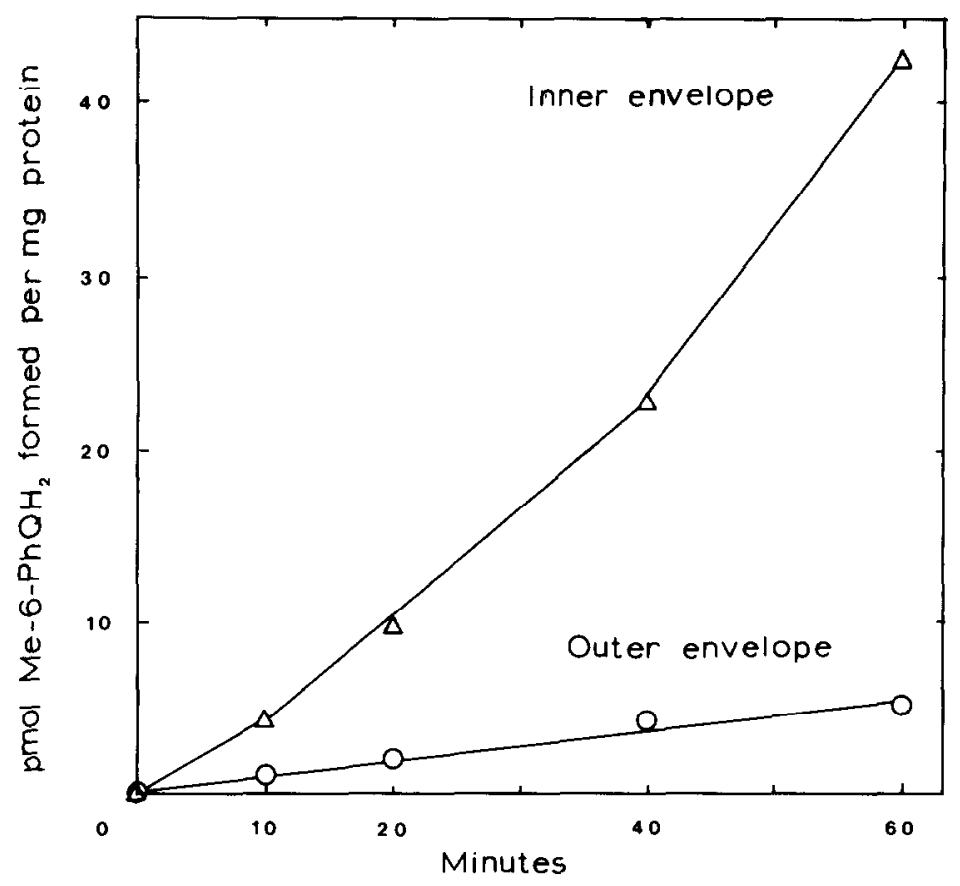

FIG. 2. Synthesis of methyl-6-phytylquinol (Me-6- $\left.\mathrm{PhQH}_{2}\right)$ in membrane fractions enriched in outer and inner envelope membranes from spinach chloroplasts. The reaction mixture, which is described under Experimental Procedures, contains $\left[{ }^{8} \mathrm{H}\right]$ homogentisic acid and phytyl-PP. 


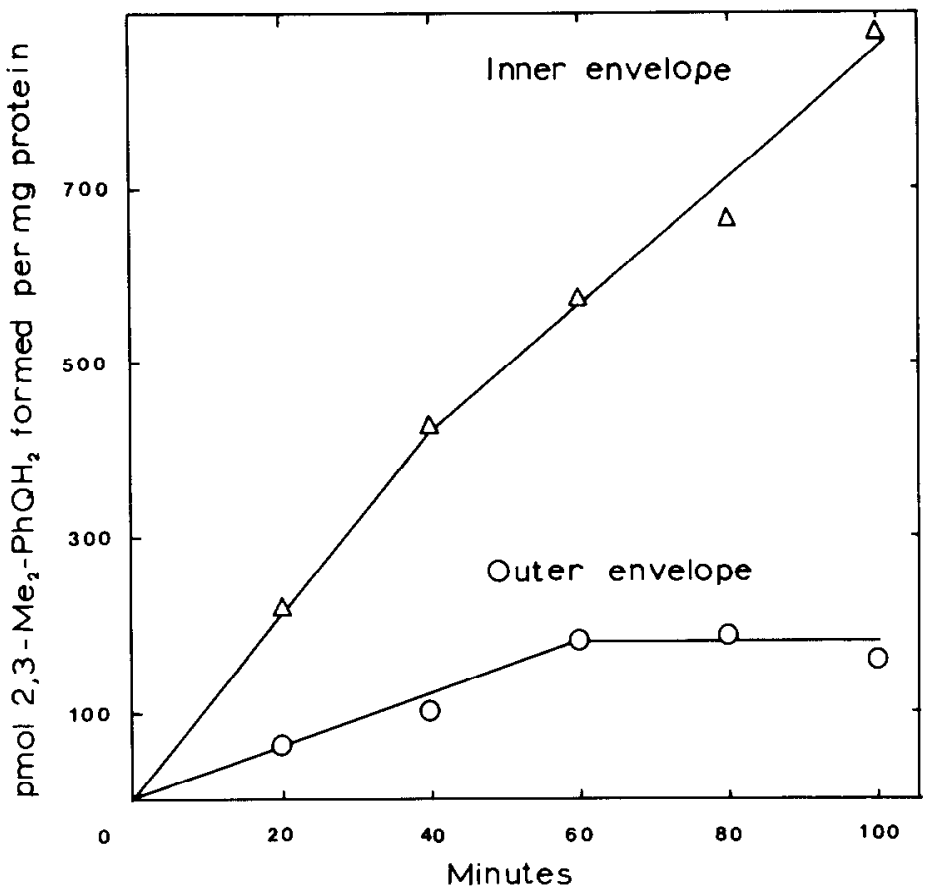

Fig. 3. Synthesis of 2,3-dimethylphytylquinol (2,3- $\left.\mathrm{Me}_{2}-\mathrm{PhQH}_{2}\right)$ in membrane fractions enriched in outer and inner envelope membranes from spinach chloroplasts. The reaction mixture, which is described under Experimental Procedures, contains methyl-6-phytylquinol and $S-\mid m e t h y l$ $\left.{ }^{14} \mathrm{C}\right]$ adenosyl-methionine.

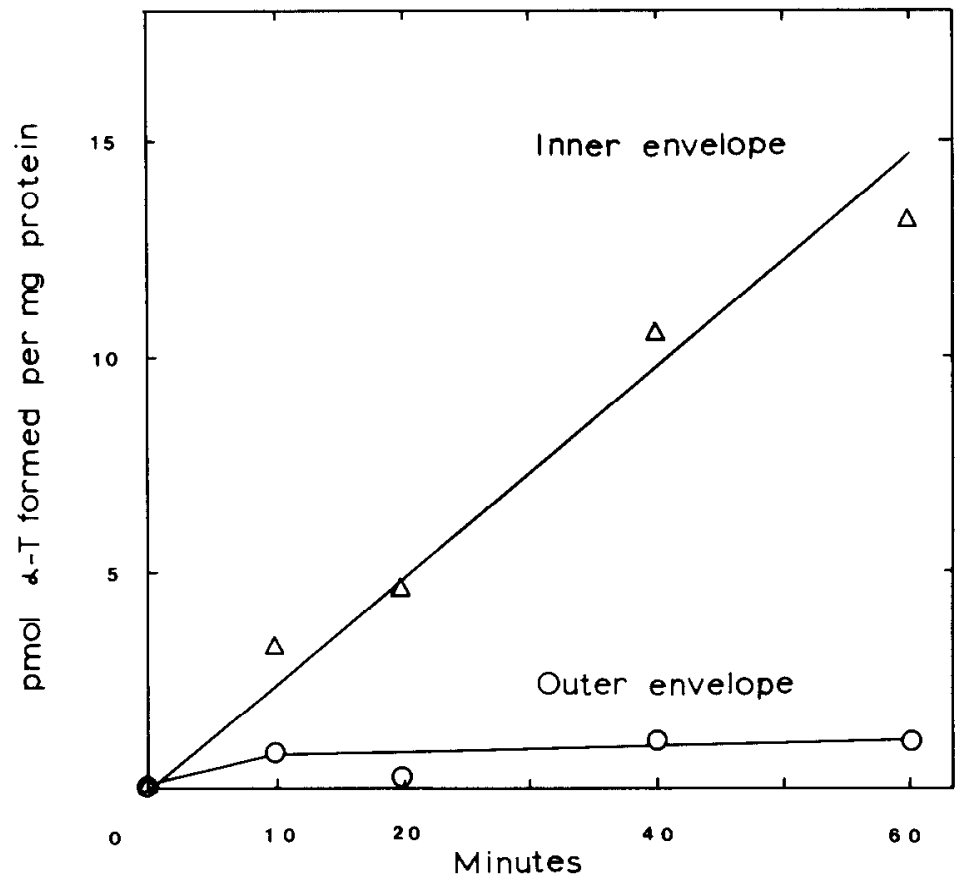

FiG. 4. Synthesis of $\alpha$-tocopherol ( $\alpha$-T) in membrane fractions enriched in outer and inner envelope membranes from spinach chloroplasts. The reaction mixture, which is described under Experimental Procedures, contains $\gamma$-tocopherol and $S-\left[\right.$ methyl- $\left.{ }^{14} \mathrm{C}\right]$ adenosyl-methionine. 
5) and they have been demonstrated in envelope membranes $(5,6)$. In fact, the reactions involved, i.e., the condensation of homogentisic acid and solanesyl-PP and the methylation of methyl-6-solanesylquinol, are similar to those involved in $\alpha$-tocopherol synthesis (compare Figs. 1 and 5). However, the intermediate (methyl6-solanesylquinol) was not available as a reference standard; therefore, we decided to assay plastoquinone synthesis in a single incubation containing the substrates which were necessary for both reactions, i.e., homogentisic acid, solanesyl-PP and $S-[$ methyl-14 C]adenosyl-methionine. As shown in Fig. 6, plastoquinone-9 synthesis occurred only in the inner envelope membrane fraction. No activity could be de- tected in the outer envelope membrane fraction.

\section{DISCUSSION}

The results presented in this article confirm and extend our previous data on the presence of prenylquinones in envelope membranes $(1,2)$ and on the localization of their synthesis in this membrane system $(5,6)$.

Both envelope membranes contain prenylquinones and in almost similar amounts, on a protein basis. We are convinced that prenylquinones are indeed genuine constituents and do not derive from contaminating thylakoids or plastoglobules for the following reasons: First,<smiles>CCCCC(C)=CCOc1ccccc1</smiles>

Homogentisic acid<smiles>CCC=C(C)CCCc1cc(O)cc(C)c1O</smiles>

2-Methyl-6-nonaprenyl-benzoquinol

$$
\begin{aligned}
& \text { SAM } \\
& \text { SAH }
\end{aligned}
$$<smiles>CCC=CCCCCCCCCCCCCC</smiles>

Plastoquinol

FIG. 5. Final steps in the formation of plastoquinone-9 in higher plant chloroplasts. 


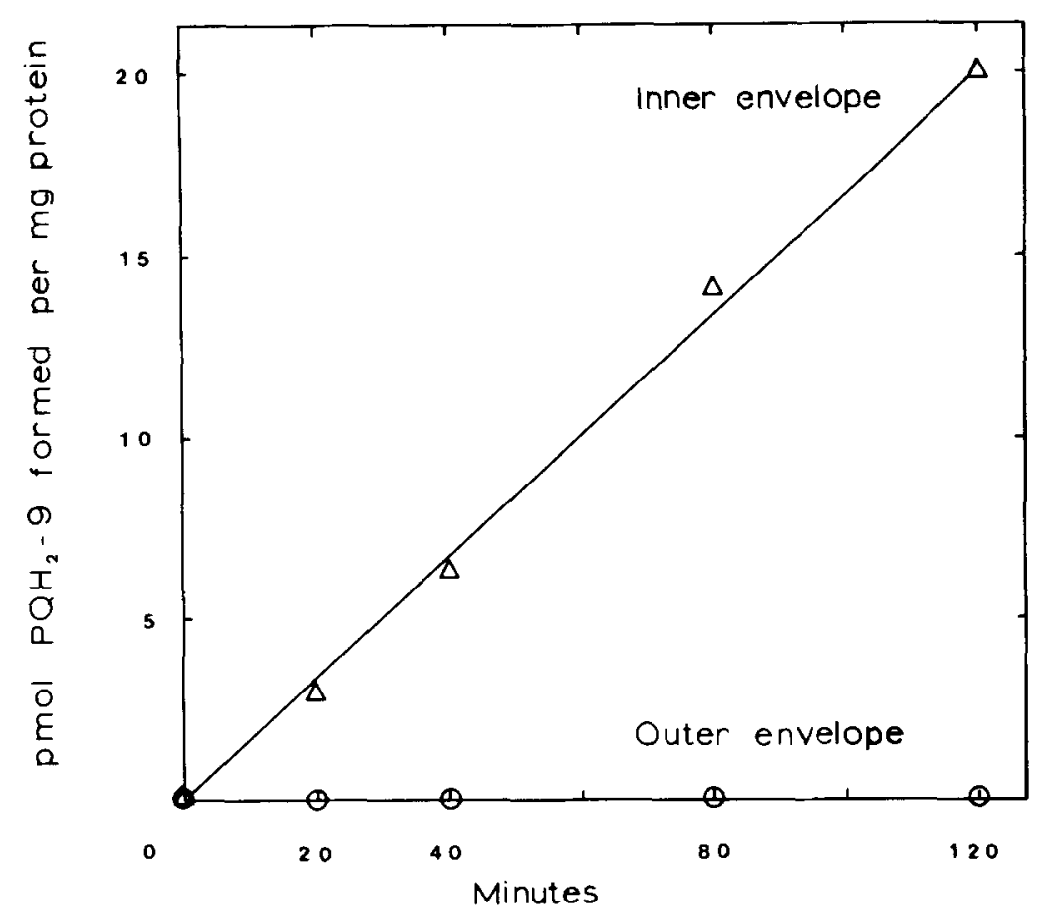

FIG. 6. Plastoquinol-9 $\left(\mathrm{PQH}_{2}\right)$ synthesis in membrane fractions enriched in outer and inner envelope membranes from spinach chloroplasts. Plastoquinol-9 formation was assayed in a reaction mixture which is described under Experimental Procedures, and contains homogentisic acid, solanesyl-PP, and $S-\left[\right.$ methyl $\left.{ }^{14} \mathrm{C}\right]$ adenosyl-methionine.

the two membrane fractions analyzed are devoid of plastoglobule clusters and of thylakoid fragments, as shown by electron microscopy (10); second, the polypeptide, polar lipid, and carotenoid composition of the two fractions $(10,11)$ and the absence of chlorophyll (10) are inconsistent with a significant contribution of prenylquinones deriving from thylakoids; and third, the prenylquinones of both envelope membranes and of thylakoids are different [see also $(1,2)$ ]. This is particularly true when we consider the $\alpha$-tocopherol to plastoquinone-9 ratios, which are $4.45,2.1$, and 0.3 for the outer envelope membrane, inner envelope membrane, and thylakoids, respectively.

However, the prenylquinone composition of the two envelope membranes is not identical. The outer envelope membrane contains more $\alpha$-tocopherol than the inner envelope membrane although this prenylquinone is the major one in both fraction; the reverse is true for plastoquinone-9, which is present in higher amounts in the inner envelope membrane than in the outer membrane. It is interesting to note that we found plastoquinone- 9 in both its oxidized and reduced forms. This is the first time that plastohydroquinone has been shown to be present in envelope membranes. However, it must be pointed out that in previous analyses $(1,2)$ lyophylized samples were analyzed; therefore, it is likely that under these conditions the reduced form of plastoquinone-9 was completely oxidized. One question which remains to be elucidated is the physiological significance of the presence of prenylquinones in envelope membranes. Indeed, the presence of both the reduced and oxidized forms of plastoquinone- 9 indicates a probable biological function for these compounds in envelope membranes. One can suggest that plastoquinone-9 might be involved in the transfer of electrons for fatty acid or carotenoid desaturation. As a matter of fact, it is now well established that envelope membranes, and especially the in- 
ner envelope membrane, play a key role in the synthesis of galactolipids [see (22) for a review]. It is, however, clear that further work is needed in order to establish the role of the envelope plastoquinone9 in fatty acids desaturation on galactolipid molecules. Another possible role for the envelope prenylquinones is as antioxidants. Indeed, the large amount of $\alpha$ tocopherol in both envelope membranes is an argument in favor of such a hypothesis. Finally, the presence of prenylquinones in envelope membranes could be the result of their biosynthesis in this membrane system. From our data, it is clear that this hypothesis is probably not entirely valid since both envelope membranes contain prenylquinones whereas only the inner one is able to synthesize these compounds.

The presence of all the enzymes involved in the last steps of plastoquinone- 9 and $\alpha$-tocopherol biosynthesis in the inner envelope membrane provides new evidence for a major role of plastid inner envelope membranes in the biogenesis of these organelles. Indeed, this membrane is not only involved in the biosynthesis of phosphatidic acid (13), diacylglycerol (13), and galactolipids $(11,13)$; from our data, we can conclude that at least five other enzymatic activities (homogentisate decarboxylase polyprenyltransferase, $S$-adenosyl-methionine:methyl-6-phytylquinol methyltransferase, $S$-adenosyl-methionine: $\gamma$-tocopherol methyltransferase, homogentisate decarboxylase solanesyltranferase, and $S$-adenosyl-methionine:methyl6-solanesylquinol methyltransferase), and possibly another one (2,3-dimethylphytylquinol cyclase) involved in the biosynthesis of the two major chloroplast prenylquinones are localized on the inner envelope membrane. However, since parts of the biosynthetic pathways for plastoquinone-9 and $\alpha$-tocopherol are almost identical (compare Figs. 1 and 5 ), it is possible that the same enzymes are involved in the biosynthesis of these two prenylquinones. The observation that prenylquinone-synthesizing enzymes are localized on the inner envelope membrane is not surprising since we have demonstrated that the soluble prenyltrans- ferase (which synthesizes prenylpyrophosphate derivatives, such as geranylgeranylpyrophosphate, from isopentenylpyrophosphate) is localized in the stromal phase and is strongly stimulated by addition of envelope membranes, and therefore probably functions in close association with the inner envelope membrane (23).

Together with our previous data $(5,6)$, these results strongly suggest that, during plastid development, massive transport of prenylquinones from the inner envelope membrane to the thylakoids must occur. Exact mechanisms are still unknown, as it is for galactolipid transport from their site of synthesis (the inner envelope membrane) to their site of accumulation (the thylakoids). A possible mechanism is the transfer via numerous vesicles which are emitted by the inner envelope membrane towards the internal membranes during plastid development [see, for instance, such figures in Ref. (24)].

Finally, these observations, together with the results obtained for galactolipid biosynthesis (11-13), demonstrate that the inner envelope membrane is a site of active membrane biosynthesis. However, more work has to be done in order to elucidate the regulation of prenylquinone biosynthesis within the inner envelope membrane and the biological function of envelope prenylquinones. These questions have major implications if we want to understand the role of each envelope membrane in plastid development.

\section{ACKNOWLEDGMENTS}

We thank I. Fisher for carrying out the HPLC analyses. This work was supported in part by the Deutsche Forschungsgemeinschaft.

\section{REFERENCES}

1. Lichtenthaler, H. K., Prenzel, U., Douce, R., and JoYaRD, J. (1981) Biochim. Biophys. Acta 641, 99-105.

2. Schultz, G., Bickel, H., BuchHolz, B., AND Soll, J. (1981) in Photosynthesis (Akoyounoglou, G., ed.), Vol. 5, pp. 311-318, Balaban International Science Services, Philadelphia.

3. Hutson, K. G., and Threlfall, D. R. (1980) Biochim. Biophys. Acta 632, 630-648. 
4. GrumbaCH, H. K., AND Forn, B. (1980) Z. Naturforsch. C 35, 645-648.

5. SOll, J., Douce, R., AND SCHultz, G. (1980) FEBS Lett. 112, 243-246.

6. Soll, J., Kemmerling, M., AND Schultz, G. (1980) Arch. Biochem. Biophys. 204, 544-550.

7. Douce, R., AND Joyard, J. (1979) Adv. Bot. Res. 7, 1-116.

8. Douce, R., Holtz, R. B., And Benson, A. A. (1973) J. Biol Chem. 248, 7215-7222.

9. Douce, R., AND Joyard, J. (1982) in Methods in Chloroplast Molecular Biology (Edelman, M., Hallick, R., and Chua, N. H., eds.), pp. 239256, Elsevier, Amsterdam.

10. Block, M. A., DoRne, A.-J., JoYARD, J., AND Douce, R. (1983) J. Biol. Chem. 258, 1327313280.

11. BLOCK, M. A., DORne, A.-J., JOYARD, J., AND Douce, R. (1983) J. Biol. Chem. 258, 1328113286.

12. Block, M. A., DORne, A.-J., JoYARD, J., AND Douce, R. (1983) FEBS Lett. 153, 377-381.

13. Block, M. A., DORNE, A.-J., JOYARD, J., AND DoUCE, R. (1983) FEBS Lett. 169, 111-115.

14. Bligh, E. G., and Dyer, W. J. (1959) Canad. J. Biochem. Physiol. 37, 911-917.
15. Threlfall, D. R., AND Goodwin, T. W. (1967) Biochem. J. 103, 573-588.

16. JoO, C. N., PARK, C. E., KRAMeR, J. K. G., AND KATES, M. (1973) Canad. J. Biochem. 51, 15271536.

17. MAYer, H., AND Isler, O. (1971) in Methods in Enzymology (McCormick, D. B., and Wright, L. D., eds.), Vol. 18, pp. 241-348, Academic Press, New York.

18. Soll, J., AND Schultz, G. (1980) Phytochemistry 19, 215-218.

19. Jeffrey, S. W, Douce, R., and Benson, A. A. (1974) Proc. Natl. Acad. Sci. USA 71, 807-810.

20. Lowry, A. R., Rosebrough, N. J., FARr, A. L., AND Randall, R. (1971) J. Biol. Chem. 193, 265-275.

21. Pennock, J. F. (1983) Biochemical Soc. Trans. 11, 504-510.

22. DOUCE, R., AND JoYARD, J. (1980) in The Biochemistry of Plants, A Comprehensive Treatise (Stumpf, P. K., ed.), Vol. 4, pp. 321-362, Academic Press, New York.

23. Block, M. A., JoYARD, J., AND DOUCE, R. (1980) Biochim. Biophys. Acta 631, 210-219.

24. Carde, J. P., Joy ARd, J., AND Douce, R. (1982) Biol. Cell. 44, 315-324. 